\title{
A Deterministic Approach to Measurement of Noise Attenuation in Oil-Rig Drill Ship Positioning Systems
}

\author{
E. C. Obinabo ${ }^{1}$, T. C. Nwaoha ${ }^{2, *}$, F. I. Ashiedu ${ }^{3}$, C. O. Izelu ${ }^{3}$ \\ ${ }^{1}$ Department of Electrical and Electronic Engineering, Ambrose Alli University, Ekpoma, Edo State, Nigeria \\ ${ }^{2}$ Department of Marine Engineering, Federal University of Petroleum Resources, Effurun, Delta State, Nigeria \\ ${ }^{3}$ Department of Mechanical Engineering, Federal University of Petroleum Resources, Effurun, Delta State, Nigeria
}

Email address:

nwaoha.thaddeus@fupre.edu.ng (T. C. Nwaoha)

*Corresponding author

\section{To cite this article:}

E. C. Obinabo, T. C. Nwaoha, F. I. Ashiedu, C. O. Izelu. A Deterministic Approach to Measurement of Noise Attenuation in Oil-Rig Drill Ship Positioning Systems. Automation, Control and Intelligent Systems. Vol. 4, No. 3, 2016, pp. 59-65. doi: 10.11648/j.acis.20160403.12

Received: May 30, 2016; Accepted: June 12, 2016; Published: June 29, 2016

\begin{abstract}
This paper presents a qualitative evaluation of wave-induced motions in an oil-rig drill ship positioning system which incorporates a priori knowledge of noise contamination in the measured data. The noise contamination $\beta$ defined in the function of the known form $(P(X, \beta))$ and $X$ takes the specific values $\mathrm{z}$, which from Cramer-Rao bound, gives the smallest possible variance with which the estimate of $\beta$ can be determined. A conceptual model of the problem based on the maximum likelihood techniques in terms of joint probability distribution functions enhanced convergence of the iteration process. A filter was postulated to define the error covariance matrix which yielded unbiased estimates of the measured data.
\end{abstract}

Keywords: Wave-Induced Motions, Oil-Rig Drill Ship, Maximum Likelihood, Error Covariance Matrix, Noise Attenuation, Filter, Minimum Variance Estimate, Error Covariance Matrix, Iteration Process

\section{Introduction}

Noise attenuation in oil-rig drill ship positioning systems has been addressed severally by recourse to filter design [1] [2] and control system modelling assuming all the noise processes are independent, and the filter designed to give a minimum variance estimate. This reduces the problem to one of optimal control whereby the best estimate using the measured values of the input and output of the system are required. Because measurements invariably contain errors [3] [4] [5]; the approach to the problem utilizes concepts of probability and statistics in which state estimation is addressed because noise is usually known to be correlated with the measured data [6] [7].

In an exploration method by acoustic means, [8] employed digital filtering and signal processing to establish a guaranteed data interpretation of the liquid level position in noisy well bores. Signal filtration in this application provides additional processing techniques under operator control to obtain accurate results in oil wells with shallow liquid levels and noisy well bores. This is enhanced by inclusion of an analogue filter [8] [9] to reduce the interference from signals generated by the reflections at the tubing collars, and to ensure that the liquid level signal could be detected in the majority of the cases. Here, the fundamental objective is markedly enhanced by increasing the signal-to-noise ratio in the recorded data. One method of doing this is to filter measured values to reject undesirably high frequency components [8] [9]. This is achieved by passing the measurements through simple low-pass filters before connecting to the input. The cut-off frequency of the filter would then be selected in conjunction with the sampling and conversion rates in order to satisfy a given sampling requirement while preserving the signal components of interest.

In digital signal processing [8] [9] [10] [11] [12], the Analogue-to-Digital Converter (ADC) gives high resolution of the data based on continuous integration and oversampling in combination with carefully designed lowpass filters (fig. 1) which eliminate gaps in the sampling process. The negative feedback from the output (fig. 1) along the 1-bit Digital-to-Analogue Converter (DAC) and 
the sum amplifier at the input are performed at a high sample rate, transferring the quantization noise into the stop band of the digital low-pass filter, for decimation. In the process, the sampled analogue signal is fed to the sum amplifier along with the output of the 1-bit Digital-toAnalogue Converter (DAC). The integrated difference signal is fed to the strobed comparator whose output samples the difference signal at the sampling frequency many times that of the analogue signal frequency. The output of the comparator provides the digital input for the 1-bit Digital-to-Analogue Converter (DAC). Thus, the system functions on a negative feedback loop which minimizes the difference signal by tracking the input. The integrator is continuously fed with the differential signal and there are no gaps in the analogue input signal as introduced by sample and hold devices. The digital information representing the analogue input voltage was coded in the polarities of the pulse train appearing at the output of the comparator which can be retrieved as a parallel binary data word applying a digital filter operator.

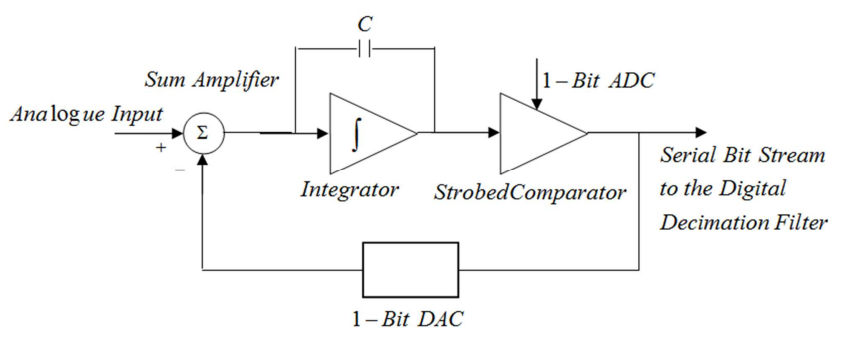

Fig. 1. A Continuous Sampling Signal Modulator [8].

The Kalman filter has received considerable attention in the existing literature [2] [13] [14] [15] [16] and has been applied in wave-induced motions of ships [17]. Given a signal model that consists of a linear dynamic system driven by stochastic white noise processes, the Kalman filter [2] [18] exploits a state space model for optimal filtration of noisy measurements. A precursor to the Kalman filter was the Weiner filter which was derived independently by Weiner and Kolmogorov [19] and which gives a method of optimally attenuating noise in process measurements. However, the Weiner filter is limited to time-invariant problems involving stationary noise sequence. The purpose of this study was to develop a statistical quality control scheme for measurements of position and heading of a ship in wave-induced motions, and to show that a filter can be postulated to define a covariance matrix to yield unbiased measurements.

\section{Formulation of the Problem}

Generally, estimation of process variables contaminated with noise is formulated on the basis of maximum likelihood [17] [2] using statistical information in terms of joint probability distribution functions. When the additive, zero-mean white Gaussian measurement noise is defined in terms of mean values and variances, which will be appropriate for many practical applications, the least- squares solution is formulated as a deterministic problem [20] [21] [22] with appropriate weighting that leads to the maximum likelihood estimate. The ship dynamics considered in this study were assumed to be completely controllable and observable. The autopilot maintains the ship on a set or desired heading while being subjected to disturbances such as wind, waves and current. The main elements of the autopilot system are shown in fig. 2. The actual heading is measured by a gyro-compass (or magnetic compass in a smaller vessel), and compared with the desired heading, dialed into the autopilot by the ship's master. The autopilot, or controller, computes the demanded rudder angle and sends a control signal to the steering gear. The actual rudder angle is monitored by a rudder angle sensor and compared with the demanded rudder angle, to form a control loop.

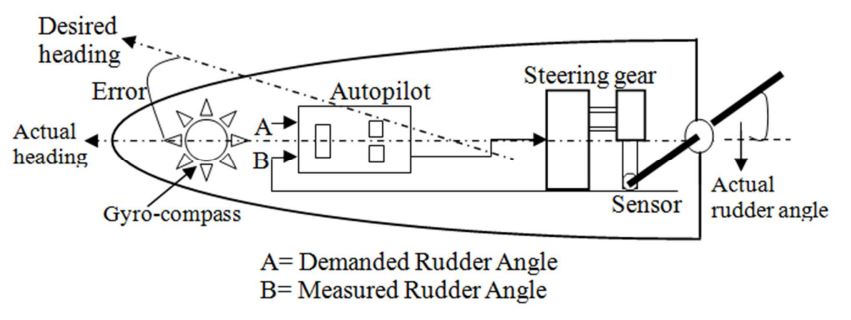

Fig. 2. Ship Autopilot Control System [23].

The rudder provides a control moment on the hull to drive the actual heading towards the desired heading while the wind, waves and current produce noise moments that may help or hinder this action. The block diagram of the system is shown in fig. 3 .

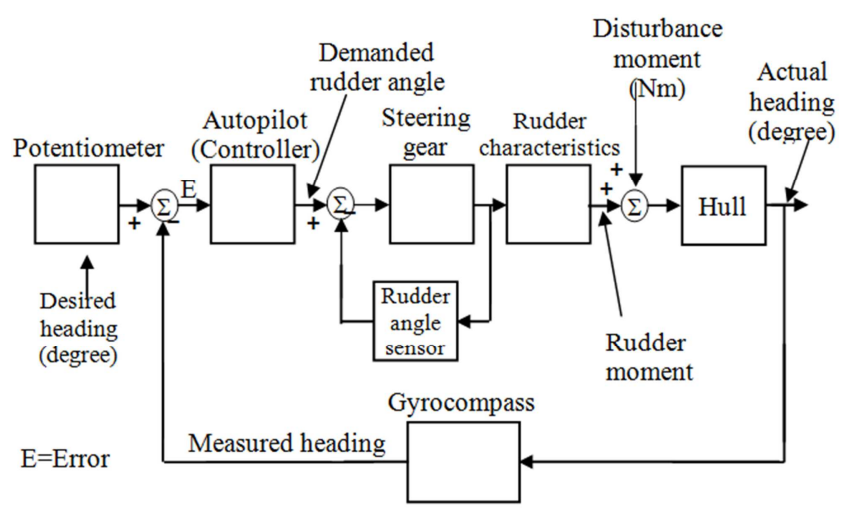

Fig. 3. Block Diagram of Ship Autopilot Control System [23].

For the purpose of this study, the ship dynamics were represented by a linear discrete-time process using the difference equation [24] [1].

$$
x(k+1)=A(k) x(k)+B(k) u(k), x(0)=x_{0}
$$

where $u(k)$ is an $r$-dimensional random vector process with the following statistical properties

$$
E[u(k)]=m(k)
$$




$$
\begin{gathered}
E\left[u\left(k_{1}\right)-m\left(k_{1}\right)\right]\left[u\left(k_{2}\right)-m\left(k_{2}\right)\right]^{T}=K\left(k_{2}, k_{1}\right) \\
E[x(k)]=\varphi(k, 0) x_{0}+\sum_{i=0}^{k-1} \varphi(k, i+1) B(i) E[u(i)]
\end{gathered}
$$

The analytical approach which often has some computational advantages is to derive a deterministic equation whose solutions are the desired mean and covariance matrix of $x(k)$. Taking the expectation of both sides of (1) yields

$$
E[x(k+1)]=A(k) E[x(k)]+B(k) E[u(k)], E[x(0)]=x_{0}
$$

This is a deterministic linear difference equation which yields as its solution the expected value of $x(k)$. To derive the covariance of $x(k)$, we subtract (5) from (1) to obtain $x(k+1)-E[x(k+1)]$ and then postmultiply both sides of the resulting equation by $[x(x+1)-E[x(k+1)]]^{T}$ and then take the expectations of both sides.

\section{Maximum Likelihood Estimate}

Estimation of the process considered in this study was based on the assumption that some or all of the parameters may be unknown even though the structure of the differential equation characterizing the system as well as the initial and boundary conditions may be available. This reduced the problem to one of optimal control whereby the best estimate using the measured values of the input and output the system are required. Because measurements invariably contain errors, solution of the problem should utilize concepts of probability and statistics in which the problem should also address state estimation because noise is usually known to be correlated with the measured data. This must be estimated at the same time as the parameters during which filtration is mandatory in the processing of the data. Computation of the optimal estimates should consequently rely on convergence of the iteration employed, which is accomplished through sequential filtration of the estimate.

We considered a time-series model of the form [25]

$$
x(j)+\sum_{i=1}^{m} \alpha_{i} x(j-i)=\sum_{i=0}^{m} b_{i} u(j-i)
$$

The partial fraction expansion required for obtaining a time solution to (6) is defined for distinct roots using the method of residues, as

$$
Y(s)=\sum_{v=1}^{k} \sum_{k=1}^{m_{\sigma}} \frac{\alpha_{v k}}{\left(s-\sigma_{v}\right)^{k}}
$$

where $\alpha_{1} \ldots \alpha_{k}$ are the poles of the function $Y(s)$ with multiplicities $m_{1} \ldots m_{k}$.

The inversion integral was then written in the form

$$
y(t)=\frac{1}{2 \pi i} \int_{\sigma-i \infty}^{\sigma+i \infty} Y(s) e^{s t} d s=\sum_{v=1}^{k} \sum_{k=1}^{m_{v}} \frac{C_{v k}}{(k-1) !} t^{k-1} e^{\sigma_{v} t}, t>0
$$

so that the function $Y(s)$ with contaminated noise becomes

$$
Y(s)=\left[\sum_{i=1}^{n} \frac{\beta_{i}}{s+\alpha_{i}}\right] U(s)+\text { white noise }
$$

where $\alpha_{i}, \beta_{i}$ are the parameters of the system, and $n$ is the order of the system

Using the shift operator $z^{-1}$ defined by $z^{-1} x(j)=x(j-1)$, equation (6) becomes

$$
y(j)=\frac{B\left(z^{-1}\right)}{A\left(z^{-1}\right)} u(k)+\lambda \frac{c\left(z^{-1}\right)}{A\left(z^{-1}\right)} e(k), k=1, \ldots, N
$$

where $y(k)$ is the observed output signal, $u(k)$ is the applied input signal, $N$ is the number of samples and $e(t)$ is the noise sequence. The polynomial operators $A, B$, and $C$ are defined as follows

$$
\begin{gathered}
A\left(z^{-1}\right)=1+\sum_{i=1}^{N A} \alpha_{i}^{\prime} z^{-i} \\
B\left(z^{-1}\right)=\sum_{i=1}^{N B} b_{i}^{\prime} z^{-i} \\
C\left(z^{-1}\right)=1+\sum_{i=1}^{N C} c_{i}^{\prime} z^{-i}
\end{gathered}
$$

If the continuous model in (4) is discretized we shall obtain a discrete time model of the form

$$
y(k)=\sum_{i=1}^{n} \frac{b_{i} z^{-1}}{1+a_{i} z^{-1}} u(k)+\lambda e(k)
$$

where the parameter set $a, b$ is related to the parameter set $\alpha, \beta$ via the relation

$$
a_{i}=-\exp \left(-\alpha_{i} T\right)
$$

and

$$
b_{i}=\frac{\beta_{i}}{\alpha_{i}}\left(1-\exp \left(-\alpha_{i} T\right)\right)
$$

where $T$ is the sampling interval in seconds. The infinite noise of the discrete observations due to aliasing that may result from the above discretization process is negligible. This is justified if $u(k)$ and $e(s)$ are independent of all $k$ and $s$. This is a reasonable assumption as long as the identification is performed for data acquired from experiments, where $u(k)$ is a priori known sequence. In some practical situations, this assumption is often violated when operating records are used because in such a case the input may depend on the output through feedback. 
The canonical model in (10) was made equivalent to the discrete-time model of (12) by satisfying the conditions

$$
\begin{aligned}
& \text { (i) } c_{i}^{\prime}=a_{i}^{\prime} \\
& \text { (ii) } \frac{B\left(z^{-1}\right)}{A\left(z^{-1}\right)}=\sum_{i=1}^{n} \frac{b_{i} z^{-1}}{1+a_{i} z^{-1}}
\end{aligned}
$$

The statistical method of maximum likelihood was employed to optimize the probability of obtaining the expected result. Consequently, a loss function $V(\theta)$ was defined as follows

$$
V(\theta)=\frac{1}{2} \sum_{k=1}^{N} \varepsilon^{2}(k)
$$

which will be minimized with respect to the system parameter set $\theta=\left[a^{\prime}, b^{\prime}\right]$. The residues were defined by

$$
\varepsilon(k)=y(k)-\frac{B\left(z^{-1}\right)}{A\left(z^{-1}\right)} u(k)
$$

so that the values of the parameter set $a^{\prime}$ and $b^{\prime}$ that make $V(\theta)$ in (15) minimum will be the estimates of the parameters of the system. The approach considered is one of finding the coefficients of the prediction model

$$
\hat{y}\left(\frac{k}{k-1}\right)=\frac{B\left(z^{-1}\right)}{C\left(z^{-1}\right)} u(k)+\frac{C\left(z^{-1}\right)-A\left(z^{-1}\right)}{c\left(z^{-1}\right)} y(k)
$$

so that the mean square prediction error

$$
V(\theta)=\sum_{k=1}^{N} y(k)-\hat{y}\left(\frac{k}{k-1}\right)^{2}=\sum_{k=1}^{N} \varepsilon^{2}(k)
$$

is as small as possible. By doing so the assumption of Gaussian distribution of the noise sequence $\varepsilon(k)$ may be relaxed. In the model given in (12), the residues were obtained as

$$
\varepsilon(k)=y(k)-\left(\sum_{i=1}^{n} \frac{b_{i} z^{-1}}{1+a_{i} z^{-1}}\right) u(t)
$$

For system order greater than $n, \varepsilon(k)$ as given in (19) becomes computationally difficult and highly nonlinear, whereas in the model of (12), the residues given by (16) may be computed quite easily for any given system order. Since the two models are equivalent via the transformations defined in (8), we are free to use any of them. Thus the form of residues given in (16) is recommended and used in this study for parameter estimation; application of a filter for signal noise attenuation becomes evident.

Now, if there exists an unknown parameter $\beta$ defined in the function of the known form $(P(X, \beta))$ and $X$ takes the specific values $z$, Cramer-Rao bound gives the smallest possible variance with which an estimate of $\beta$ can be determined. Now, let $\hat{g}(z)$ be an unbiased estimate of the given function $g(\beta)$. Since it is unbiased, we write

$$
E[\hat{g}(z)]=g(\beta)
$$

i.e.,

$$
\int_{-\infty}^{+\infty} \hat{g}(z) P(z) d x=g(\beta)
$$

Since $z$ was drawn from the process which generated $X$, it has the same probability density function

$$
p(z)=\left.P(X, \beta)\right|_{x=z}=L(z, \beta)
$$

Re-writing (12)

$$
\int_{-\infty}^{+\infty} \hat{g}(z) L(z, \beta) d z=g(\beta)
$$

Differentiating with respect to $\beta$ gives

$$
\int_{-\infty}^{+\infty}\left(\hat{g}(z) \frac{\partial L}{\partial \beta} d z\right)=g^{\prime}(\beta)=\frac{d g}{d \beta}
$$

Since $L(z, \beta)$ is a probability function

$$
\int_{-\infty}^{+\infty} L(z, \beta) d z=1
$$

and its differential with respect to $\beta$ is zero, that is,

$$
\int_{-\infty}^{+\infty} \frac{\partial L}{\partial \beta} d z=0
$$

then

$$
\int_{-\infty}^{+\infty} g(\beta) \frac{\partial L}{\partial \beta} d z=0
$$

Substituting (21) from (24) gives

$$
\int_{-\infty}^{+\infty}[\hat{g}(z)-g(\beta)] \frac{\partial L}{\partial \beta} d z=g^{\prime}(\beta)
$$

We now consider the log-likelihood function as follows:

$$
\begin{gathered}
L(z, \beta)=\log _{e} L(z, \beta) \\
\frac{\partial L}{\partial \beta}=\frac{1}{L} \frac{\partial L}{\partial \beta}
\end{gathered}
$$

Substituting in (25) gives 


$$
\int_{-\infty}^{+\infty}[\hat{g}(z)-g(\beta)] L \frac{\partial L}{\partial \beta} d z=g^{\prime}(\beta)
$$

Now from, the Schwartz inequality

$$
\left\{\int_{-\infty}^{+\infty}[\hat{g}(z)-g(\beta)]^{2} L d z\right\}\left\{\int_{-\infty}^{+\infty}\left(\frac{\partial L}{\partial \beta}\right)^{2} L d z\right\} \geq\left(g^{\prime}(\beta)\right)^{2}
$$

where

$$
\left\{\int_{-\infty}^{+\infty}[\hat{g}(z)-g(\beta)]^{2} L d z\right\}=\operatorname{Var} \hat{g}(z)
$$

and

$$
\left\{\int_{-\infty}^{+\infty}\left(\frac{\partial L}{\partial \beta}\right)^{2} L d z\right\}=E\left[\left(\frac{\partial L}{\partial \beta}\right)^{2}\right]
$$

Thus for any unbiased estimate the lower bound on the variance of the estimate is given by Cramer-Rao bound as follows:

$$
\operatorname{Var} \hat{g}(z) \geq \frac{\left[g^{\prime}(\beta)\right]^{2}}{E\left[\left(\frac{\partial L}{\partial \beta}\right)^{2}\right]}
$$

If $\hat{g}(z)$ is an unbiased estimate of $\beta$, i.e., $g(\beta)=\beta$

Therefore

$$
\begin{gathered}
g^{\prime}(\beta)=\frac{d g}{d \beta}=1 \\
\operatorname{Var}[\hat{\beta}] \geq \frac{1}{E\left[\left(\frac{\partial L}{\partial \beta}\right)^{2}\right]}
\end{gathered}
$$

\section{Application}

In an oil-rig drill ship positioning system which incorporates a priori knowledge of noise contamination in the measured data, it is required to obtain an estimate of the mean value of the position of the ship based on independent measurements $X\left(x_{1}, x_{2}, \ldots, x_{N}\right)$ with the time between successive data acquisition to be distributed with a probability function of the form

$$
P\left(X ; Q_{1}, Q_{2}\right)=\frac{1}{Q_{1} \sqrt{2 \pi}} \exp \left[\frac{1}{2}\left(\frac{x-Q_{1}}{Q_{2}}\right)^{2}\right]
$$

which describes the probability that the parameters $Q_{1}$ and $Q_{2}$ caused the measurements $X\left(x_{1}, x_{2}, \ldots, x_{N}\right)$ to occur.

Here, we obtained an estimate of the mean position of the ship and expressed it as a product of the individual functions:

$$
L\left(Q_{1}, Q_{2}, \ldots / x_{1}, x_{2}, \ldots, x_{N}\right)=\prod_{i=1}^{N} L\left(Q_{1}, Q_{2}, \ldots / x_{i}\right)
$$

$$
\begin{gathered}
=\left\{\frac{1}{Q_{2} \sqrt{2 \pi}} \exp \left[\frac{1}{2}\left(\frac{x_{1}-Q_{1}}{Q_{2}}\right)^{2}\right]\right\}\left\{\frac{1}{Q_{2} \sqrt{2 \pi}} \exp \left[\frac{1}{2}\left(\frac{x_{2}-Q_{1}}{Q_{2}}\right)^{2}\right]\right\} \ldots \\
=\frac{1}{\left(Q_{2} \sqrt{2 \pi}\right)^{N}} \exp \left[\frac{1}{2} \sum_{i=1}^{N}\left(\frac{x_{i}-Q_{1}}{Q_{2}}\right)^{2}\right]
\end{gathered}
$$

For covariance, we define the log-likelihood function

$$
\begin{gathered}
L\left(Q_{1}, Q_{2}, \ldots / x_{1}, x_{2}, \ldots, x_{N}\right)=\log _{e}\left(Q_{1}, Q_{2}, \ldots, / x_{1}, x_{2}, \ldots, x_{N}\right) \\
=\log _{e} L=-N \log _{e}\left(Q_{2} \sqrt{2 \pi}\right)-\frac{1}{2} \sum_{i=1}^{N}\left(\frac{x_{i}-Q_{1}}{Q_{2}}\right)^{2}
\end{gathered}
$$

which we maximized with respect to $Q_{1}, Q_{2}$ by equating to zero the partial derivatives of $L$ as follows:

$$
\frac{\partial L}{\partial Q_{1}}=\frac{\partial \sum_{i=1}^{N} \log _{e} P\left(x_{i ;} Q_{1}, Q_{2}, \ldots\right)}{\partial Q_{1}}=0
$$

and

$$
\frac{\partial L}{\partial Q_{2}}=\frac{\partial \sum_{i=1}^{N} \log _{e} P\left(x_{i ;}, Q_{1}, Q_{2}, \ldots\right)}{\partial Q_{2}}=0
$$

Maximizing with respect to $Q_{1}$ and $Q_{2}$ gives

$$
\begin{aligned}
& \frac{\partial L}{\partial Q_{1}}=\frac{1}{Q_{2}^{2}} \sum_{i=1}^{N}\left(x_{i}-Q_{1}\right) \\
& \frac{\partial L}{\partial Q_{2}}=\frac{N}{Q_{2}}+\frac{1}{Q_{2}^{2}} \sum_{i=1}^{N}\left(x_{i}-Q_{1}\right)^{2}
\end{aligned}
$$

Letting $\frac{\partial L}{\partial Q_{1}}=\frac{\partial L}{\partial Q_{2}}=0$ gives the maximum likelihood of the estimates as follows

$$
\begin{aligned}
& \hat{Q}_{1}=\frac{1}{N} \sum_{i=1}^{N} x_{i}=\bar{x} \\
& \hat{Q}_{2}=\left(\frac{1}{N} \sum_{i=1}^{N}\left(x_{i}-\bar{x}\right)^{2}\right)^{\frac{1}{2}}
\end{aligned}
$$

From the Cramer-Rao bound 


$$
\operatorname{Var}(\hat{\beta})=-\frac{1}{E\left(\frac{\partial^{2} L}{\partial \beta^{2}}\right)}
$$

we obtained, for $Q_{1}$,

$$
\frac{\partial^{2} L}{\partial Q_{1}^{2}}=-\frac{N}{Q_{2}^{2}}
$$

so that

$$
\operatorname{Var}\left[\hat{Q}_{1}\right] \geq-\frac{1}{\left(-\frac{N}{Q_{2}^{2}}\right)}=\frac{Q_{2}^{2}}{N}
$$

showing that the estimate is consistent with $N \rightarrow \infty$ as $\operatorname{Var}\left[\hat{Q}_{1}\right] \rightarrow 0$.

The wave-induced motions of a ship considered in this paper was concerned essentially with measurements which are correlated with coloured noise assumed to be additive to the output.

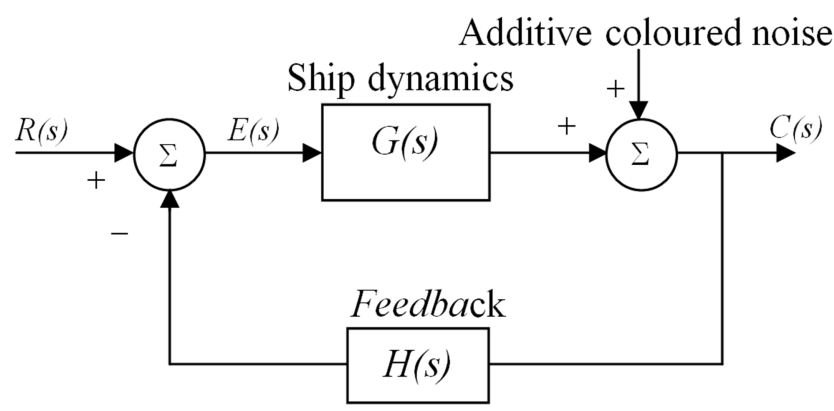

Fig. 4. Block Diagram of Ship Dynamics System.

The modelling of (19) was enhanced using the discretetime stochastic sequence as follows

$$
y(k)=\frac{B z^{-1}}{A z^{-1}} u(k)+\frac{D z^{-1}}{C z^{-1}} \eta(k)
$$

Multiplying through (50) by $A z^{-1}$ :

$$
A y=B u+\frac{A D}{C} \eta
$$

i.e.,

$$
A y=B u+e
$$

where $e=A D C^{-1} \eta(k)$ is the correlated noise sequence (53)

In matrix form we write (50) as follows

$$
\gamma=\varphi \beta+e
$$

Using least squares:

$$
\begin{gathered}
\hat{\beta}=\left(\varphi^{T} \varphi\right)^{-1} \varphi^{T} \gamma \\
=\left(\varphi^{T} \varphi\right)^{-1} \varphi^{T}(\varphi \beta+e) \\
=\beta+\left(\varphi^{T} \varphi\right)^{-1} \varphi^{T} e \\
\varphi^{T} \varphi(\hat{\beta}-\beta)=\varphi^{T} e
\end{gathered}
$$

Taking the expected values of both sides of (50) gives:

$$
E\left[\varphi^{T} \varphi(\hat{\beta}-\beta)\right]=E\left[\varphi^{T} e\right]
$$

Since $y(k)$ depends on $e(k)$ then $\varphi=[u: y]$ and $e$ are correlated.

Thus

$$
E\left[\varphi^{T} e\right] \neq 0
$$

Therefore the least square estimates of $\hat{\beta}$ are biased.

From (55),

$$
E[\hat{\beta}]=\beta+\left(\varphi^{T} \varphi\right)^{-1} E\left[\varphi^{T} e\right]
$$

An unbiased estimate of $\beta$ can only be obtained if the noise sequence $e(k)$ is reduced to a white noise sequence. In order to reduce the noise sequence to a white noise sequence, we considered (51) and postulated a filter $F$ [19] [17] such that

$$
F\left(z^{-1}\right)=1+f_{1} z^{-1}+f_{2} z^{-1}+\ldots
$$

We then obtained:

$$
A(F y)=B(F u)+F\left(\frac{A D}{C}\right) \eta
$$

Now, we chose $F=\frac{C}{A D}$ so that (61) becomes:

$$
A(F y)=B(F u)+\eta
$$

thereby reducing the additive noise $e\left(=\frac{A D}{C} \eta\right)$ to a white noise sequence $\eta$. The least square estimate $\hat{\beta}$ is now unbiased. $F u$ and $F y$ are filtered inputs and outputs.

$$
u^{F}=F u ; y^{F}=F y
$$

\section{Conclusions}

A stochastic time-invariant model has been developed for linear filtering and prediction of state in wave-induced motions of a ship. The model provides a processing technique under 
operator control for eliminating the interference from signals generated by the wave-induced motion. The result presented has shown that the wave-induced motions of an oil-rig drill ship positioning system which incorporates a priori knowledge of noise contamination in the measured data can be estimated using the maximum likelihood techniques in terms of joint probability distribution functions. The ship dynamics itself is represented by a stable, linear, parameter-dependent state model.

\section{References}

[1] Grimble M. J. (1981). Adaptive Kalman Filter for Control Systems with Unknown Disturbance IEE Proc. 128, pt. D, 6, 263-267.

[2] Kalman, R. E. (1960). A new approach to linear filtering and prediction problems, Trans. ASME J. Basic Eng., 82, 35-45.

[3] Ekejiuba, A. O. M. (1986). Locating By-passed Hydrocarbon Accumulation in Old Reservoirs, Nigerian Association of Petroleum Explorationists, Fourth Annual Conference.

[4] Ahonsi, B. and Gdula, J. (1993). A Case History of By-passed Oil in the Niger Delta, Society of Petroleum Engineers, Nigeria Council, 35-46.

[5] Weber, K. J. (1971). Sedimentological Aspects of Oil Fields in the Niger Delta, Goelogie en Mijnbouw Sc., 559-576.

[6] Dalley, R. M., Gevers, E. C. A. Stamfli, G. M. Davies, D. J. Gastaldi, C. N. Ruijtenberg, P. R. and Vermeer, G. I. O. (1989). DAZIL (Dip Azimuth, Illumination), First Break, 7 (3), 86-100.

[7] Esho, K. M. (1993). Prediction of Remaining Oil in the Imo River D2.0 Sand Using 3D Seismic Data, Society of Petroleum Engineers, Nigeria Council, 23-28.

[8] Van Wagoner, J. C., Mitchum, R. M. K. Campion, M. and Rahmanian, V. D. (1990). Siliciclastic Sequence Stratigraphy in Well Logs, Core and Outcrops: Concept for HighResolution Correlation of Time and Facies, AAPC Methods in Exploration Series, No. 7.

[9] Weber, K. J. (1986). Hydrocarbon Distribution Patterns in Nigeria Growth Fault Structures Controlled by Structural Style and Stratigraphy, Journal of Petroleum Science and Engineering, 1, 91-104.

[10] Benato, G., D'Andrea, V., Cattadori, C., and Riboldi, S. (2015). Improvement of the GERDA Ge detectors energy resolution by an optimized digital signal processing, Physics Procedia, 61, 673-682.

[11] Lépy, M. C., Cissé, O. I., and Pierre, S. (2014). Comparison of digital signal processing modules in gamma-ray spectrometry" Applied Radiation and Isotopes, 87, 402-406.
[12] Seyfried, D., Brueckner, S., and Schoebel, J. (2014). Comparison of antenna dispersion and digital signal processing effects in ultrawideband ground penetrating radar systems, Journal of Applied Geophysics, 101, 20-26.

[13] Hsiao C. H. and Wang, W. J. (2000). State Analysis and Parameter Estimation of Bilinear Systems Via Haar Wavelets, IEEE Trans. on Circuits and Systems 1: Fundamental Theory and Applications, 47 (2), 246-250.

[14] Hong, L., Girsang, I. P., Dhupia, J. S. (2016). Identification and control of stick-slip vibrations using Kalman estimator in oil-well drill strings, Journal of Petroleum Science and Engineering, 140, 119-127.

[15] Tan, C., Dai, W., Yeung, H., and Dong, F. (2015). A Kalman estimation based oil-water two-phase flow measurement with CRCC, International Journal of Multiphase Flow, 72, 306317.

[16] Liu, M., Lai, J., Li, Z., and Liu, J. (2016). An adaptive cubature Kalman filter algorithm for inertial and land-based navigation system, Aerospace Science and Technology, 51, 52-60.

[17] Athans, M. (1971). The role and use of the stochastic linear quadratic Gaussian problem in control system design, IEEE Trans. Automatic Control AC-16, 529-552.

[18] Foster, M. R. and Saffman, P. G. (1970). The Drag of a Body Moving Transversely in a Confined Stratified Fluid, Journal of Fluid Mech., 43 (2), 407 - 418.

[19] Obinabo, E. C. and Ojieabu, C. E. (2010). Measurement Noise Filtration and State Estimation of a Discrete-time Stochastic Process, International Journal of Soft Computing, 5 (2), 29-34 .

[20] Bacher, R. A., Gray, W. and Murray-Smith, D. J. (1981). Time Domain System Identification Applied to Non - invasive Estimation Cardiopulmonary Quantities ibid, 128 (2), 56-64.

[21] Ho Y. C. (1962). On the Stochastic approximation method and optimal filtration theory. Journal Math anal Applications, 6, $152-154$.

[22] Sage A. P. (1967). Least Squares Curve Fitting and Discrete Optimum Fitting, IEEE Trans. EDUC. E-10, 1, 29-38.

[23] Obinabo, E. C. (2015). The Development of Oil-rig Drill Ship Positioning Systems, Bowens Nigeria Limited, Warri, Nigeria, 425.

[24] Ljung I. (1979). Asymptotic behaviour of the extended Kalman filter as a parameter Estimation for linear Systems, IEEE Trans, ac-24, 36-50.

[25] Kochhar, A. K. (1978). Comparison of Stochastic Identification Techniques for Dynamic Modelling of Plastic Extrusion Processes, Proc. Instn. Mech. Engrs., 192 (28), 299 309. 